\title{
2 A new fuzzy de novo programming approach for optimal system design
}

\begin{abstract}
De novo programming, which evaluates the concept of optimality from a different point of view, makes it possible to rearrange the number of resources for constraint functions depending on the budget constraint. While the resource amounts of the constraints are used at full capacity, this arrangement improves the performance level of the objective functions. With the development of fuzzy set theory, mathematical programming models can be examined in a fuzzy environment as in other scientific fields. Mathematical programming models are handled in different ways as fuzzy decision variable, fuzzy goal, and fuzzy parameter. The fuzzy goal and fuzzy parameter models are used in the literature for de novo programming, also known as optimal system design. In this study, a new fuzzy de novo programming approach was proposed by using positive and negative ideal solutions. In the proposed approach, using fuzzy unit prices of resources and fuzzy resource amounts of constraints, the fuzzy budget was created. The solution steps of the developed approach are given step by step on an illustrative example accepted in the literature.
\end{abstract}

Keywords: Multiobjective de novo programming, multiobjective linear programming, fuzzy de novo programming, optimal system design

\subsection{Introduction}

In classical linear programming (LP) problems, there is a goal to be achieved based on certain decision variables and constraints. The solution of such problems determines the decision variable values as well as the objective function values based on them. On the other hand, it is possible to define the resource amount in each constraint using the values of decision variables. Instead of focusing on objective function values after solving the problem, it may answer significant problems to concentrate on the utilization amount of constraint resources, such as "Are resource amounts utilized optimally?" The answer to this problem would result in the interpretation of all the functions in the problem. When a problem is analyzed in terms of the amount of resource utilization, it mostly leads to an excess amount of resources and/ or a need for more. Either of them is a reason that hinders the optimal utilization of resources for production and also causes a downfall in the performance level of objective function. All constraints must be active for classical LP to determine an

Nurullah Umarusman, Faculty of Economics and Administrative Sciences, Aksaray University, Turkey

Ә Open Access. () 2020 Nurullah Umarusman, published by De Gruyter. (c) BY-NC-ND This work is licensed under a Creative Commons Attribution-NonCommercial-NoDerivatives 4.0 International License.

https://doi.org/10.1515/9783110628616-002 
optimal result. In other words, all constraint resources must be utilized at full capacity. Then, such a problem is named as an optimal model in terms of resource utilization. In multiobjective linear programming (MOLP) problems, the conditions described above are taken to a different dimension with "the increase in the number of goal function." It is because each goal function is achieved at different values of different variables in the solution of MOLP problems. That would lead to differences in resource utilization amounts of constraints in terms of each objective function.

It is an expected case in MODM problems that constraints have limited amounts and goal functions are not homogeneous. Therefore, there is no concept of "optimization” in MODM (multiple objective decision-making) problems. That is why it is almost impossible for all goal functions to be realized optimally and simultaneously [1]. A significant feature of MODM methods is the concept of "optimality," which is defined for single objective optimization problems but not valid for MODM problems. Instead of the concept of "optimality," decision makers therefore use the concept of paretooptimality (alternatively, efficient, nondominated, or noninferior), proposed by Italian economist Pareto, for MODM problems [2]. The concept of "Pareto-optimality" is named as "pareto-optimal” solutions for MODM problems as defined by $\mathrm{Yu}$ [3]. The set number of nondominated solutions is quite high in MOLP problems. In a MODM problem with p-number of goal functions, the concept of "optimality" for any goal function may not be applicable to other p-1 goal functions. The fundamental process in evaluating nondominated solutions is how to determine the proximity to ideal solution points (Cohon, 1978). The methods in MODM classification are used in the solution of MOLP problems. It is possible to reach a satisfactory solution among nondominated solutions by using the interactive methods such as step method, Geoffrion-Dyer-Freinberg method, and Zoints and Wallenius method [4]. Another process in evaluating nondominated solutions is the analysis based on positive and negative ideal solutions. The utility approach, goal programming, interactive approaches, and fuzzy approach models can be applied to reach a compromise solution [5]. Additionally, Tabocanon (1988) described solutions of MOLP problems using global criterion method, interactive methods, and compromise programming methods.

The studies by Zeleny $[6,7]$ provided a different viewpoint in solving single objective linear programming (SOLP)/MOLP problems. Named as "optimal system design," this approach enables the reorganization of resource amounts using "budget." Also known as de novo programming, "optimal system design" can be regarded as a special extension of LP. The most significant feature of de novo programming is that it can reorganize resource amounts of constraints based on the budget constraint. Resource amounts of constraints are utilized at full capacity with this reorganization. It brings an optimal model in terms of resource utilization. Therefore, de novo programming approach may be named as a redesigned SOLP/MOLP model. "De novo" hypothesis may be easily applied to MOLP problems as well. However, "optimal system design” application for MOLP inhabits a long process. 
This study proposes a new approach for solutions of de novo programming problems in fuzzy environment. This approach is built on the approach by Li and Lee [8], which uses positive and negative ideal solutions. The proposed approach is analyzed with an illustrative example.

\subsection{An overview of the multiobjective de novo programming process}

The first study about de novo programming, which designs an optimal system instead of optimizing a given system, was carried out by Zeleny [6]. Today's production and management systems are necessarily becoming more and more flexible. They need to be designed and redesigned rapidly, disassembled and reassembled again, which requires continuous reorganization of resources for ensuring optimality [9]. As all systems are created within their limits, they lack alternatives, options, and design variables in their creation environment. Therefore, when a system is to be redesigned, reconfigured, or optimized, its limitations and boundaries have to be done so as well. It is not sufficient to reconfigure it based on a given system with its own priorities and options. Therefore, system design requires creations of alternatives instead of selection [10]. In contrary to optimizing a given system as standard approaches do, de novo enables constraint variables and works to reach a solution that is more reassuring than those within the system's limitations. This approach must be implemented prior to the onset of production because optimal production is necessarily dependent on optimal utilization of raw materials which is only possible with an optimal production plan (Babic and Pavic, 1996).

\subsubsection{De novo programming literature survey}

It is possible to classify the scientific articles on de novo programming based on their theoretical and application aspects. This subtitle summarizes the studies that theoretically contribute to de novo programming problems.

As stated above, Zeleny [6] was the first to propose de novo programming approach, which designs an optimal system. Zeleny [7] applied to a classical LP problem. These two studies make up the foundation of de novo programming problem. Zeleny [11] summarized the approach and methodology of de novo programming, which is related to extensive LP and LP-related problems. Implications for solving traditional LP problems are also given in his study. It also provided new knowledge on the "new concept" of optimality and of MCDM. Hessel and Zeleny [12] used the concept of metaoptimum to explain how the system design 
can be carried out for multiobjective de Novo programming. Zeleny (1989) proposed an ERA (External Reconstruction Approach) method with very simple philosophy. The right side constants of constraints are organized using its specific algorithm. This organization enables the full utilization of constraint constants. It can be regarded as a different aspect of de novo approach. This approach does not use the budget constraint. Zeleny [10] suggested the "optimal path method" to create the design of an optimal system to conduct the solution of a de novo problem through an ideal system design. Shi [13] proposed in his study that several optimum path ratios could be used as alternatives to select the optimal system design to solve multiobjective de novo programming problems. Apart from "optimum path ratios" proposed by Zeleny [10] and Shi [13], there is no certain method to be used in solving multiobjective de novo programming problems. Some studies make use of MODM methods to solve multiobjective de novo programming problems. Tabocanon (1988) solved multiobjective de novo programming problems by using global criterion method, compromise constraint method, and step method. Umarusman [14] demonstrated how multiobjective de novo programming problems could be solved by using minmax goal programming. Umarusman and Türkmen [14] suggested the global criteria method, which relies on positive ideal solutions to solve multicriteria de novo programming problems. Zhuang and Hocine [15] proposed the meta goal programming for the solutions of multicriteria de novo programming problems. Banik and Bhattacharya (2018) suggested weighted goal programming method to solve multiobjective de novo programming problems.

The short literature survey above lists some of the articles that investigate the solution of multiobjective de novo programming problems in classical environments. In addition to classical decision-making process, there are significant studies on de novo programming problems in fuzzy environments as well. Below are the theoretical studies on the de novo programming in fuzzy environments. First, Lai and Hwang [16] made use of Chanas' (1983) nonsymmetric approach to solve a single criterion de novo programming problem. Li and Lee [17] were the first to propose a fuzzy approach to solve a multicriteria de novo programming problem using "the possibility concept of fuzzy set theory.” In their study as a continuation of the former, Li and Lee [18] suggested a "two-step fuzzy approach" based on positive ideal solutions and negative ideal solutions. They proposed that their approach is very efficient and can be applied to most multicriteria de novo programming problems. In their next article which is considered to be a continuation of their first study where they analyzed multicriteria de novo programming in a fuzzy logic frame, Li and Lee [8] studied fuzzy goals and fuzzy coefficients simultaneously and proposed a different approach. Bhattacharya and Chakraborty [19] suggested an alternative method for general multiobjective de novo programming problems under fuzzy environment, which uses Luhandjula's compensatory $\mu \_\theta$-operator. Banik and Bhattacharya (2018) put forward a one-step method to solve multiobjective de novo programming problem, which makes use of min-max goal programming technique where all parameters are fuzzy numbers. Umarusman 
[20] suggested for solving multiobjective de novo programming problem in fuzzy setting using $\mathrm{Wu}$ and Guu (2001) approach.

\subsubsection{Multiobjective de novo programming formulation}

Similar to MOLP problems, multiple objective de novo programming problems are handled with more than one and conflicting objective functions based on the same constraint functions. Multiple objective de novo programming is demonstrated mathematically as follows [10]:

$$
\begin{aligned}
& \operatorname{Max} Z_{k}: \sum_{j=1}^{n} C_{k j} x_{j} \\
& \operatorname{Min} W_{s}: \sum_{j=1}^{n} C_{s j} x_{j}
\end{aligned}
$$

Subject to

$$
\begin{aligned}
& \sum_{j=1}^{m} A_{j} X_{j} \otimes B \\
& x_{j} \geq 0, \quad k=1,2, \ldots, l, s=1,2, \ldots, r, \text { and } j=1,2, \ldots, n
\end{aligned}
$$

where

$A_{j}=\sum_{i=1}^{m} p_{i} a_{i j}$ is the total budget with $B$ utilization, and $P_{i}$ is the unit price of $i$ th resource. $\otimes$ symbol means that the budget constraint is " $\leq$ " or "=" type. This decision rests on the decision maker. "=" allows the total use of budget constraint, whereas " $\leq$ " means that it should be less than the current budget.

De novo formulation provides not only the best mixture of outputs but also the best mixture of the inputs (Tabucanon, 1988). One of the advantages of this approach is that it enables the adjustment of resource constraints in multicriteria decision-making problems and that it makes the initial solution more convenient at the same or less cost. Resource amounts of constraints are utilized at full capacity with de novo solution. It enables slack, surplus, and artificial variables to be equal to zero. When the solution of de novo programming problems is investigated from MOLP perspective, positive and negative ideal solutions [21] must be determined for each objective functions. The positive ideal solution set is indicated as $I^{*}=\left\{Z_{1}^{*}, Z_{2}^{*}, \ldots, Z_{l}^{*} ; W_{1}^{*}, W_{2}^{*}, \ldots, W_{s}^{*}\right\}$ and negative ideal solution set as $I^{-}=\left\{Z_{1}^{-}, Z_{2}^{-}, \ldots, Z_{l}^{-} ; W_{1}^{-}, W_{2}^{-}, \ldots, W_{s}^{-}\right\}$. Then the solution of eq. (2.1) may be done in terms of "optimal path ratio" as proposed by Zeleny [10] and Shi [13], based on positive and negative ideal solutions. 


\subsection{An approach proposal for fuzzy multiobjective de novo programming}

Conventional optimization methods evaluate parameters based on precise data. However, decision makers want to know which changes in parameters would result in which changes in the solution of the problem. It is because the precise parameters used by decision makers are only valid for "that moment." It becomes harder to determine optimal values of goals when short- and long-term changes in parameters are accounted for. The development of fuzzy set theory by Zadeh [22] and further improvements made it possible to acquire more flexible and informative results that present solution by forming given parameters structured as fuzzy ranges. Fuzzy set theory, which helps decision makers in fuzzy decision environments, may be easily applied to multiobjective de novo programming problems. This chapter presents a new methodology based on the proposal by Li and Lee [8], which accounts for both goals and coefficients at the same time.

The model by Li and Lee [8], which presents fuzzy goals and fuzzy coefficients $\left(\tilde{c}_{k j}, \tilde{c}_{s j}, \tilde{a}_{i j}, \tilde{p}_{i}\right.$, and $\left.\tilde{B}\right)$ formed by using positive ideal and negative ideal solution information is here solved as a two-step fuzzy approach. Consider the fuzzy multicriteria de novo programming problem:

$$
\begin{aligned}
& \operatorname{Max} \tilde{Z}_{k}(x)=\sum_{j=1}^{n} \tilde{c}_{k j} x_{j} \\
& \operatorname{Min} \tilde{W}_{s}(x)=\sum_{j=1}^{n} \tilde{c}_{s j} x_{j}
\end{aligned}
$$

Subject to

$$
\begin{aligned}
& \sum_{j=1}^{n} \tilde{a}_{i j} x_{j}-b_{i} \leq 0 \\
& \sum_{i=1}^{m} \tilde{p}_{i} b_{i}=\tilde{B} \\
& x_{j} \geq 0, i=1,2, \ldots, m, j=1,2, \ldots, n, k=1,2, \ldots, l, \text { and } s=1,2, \ldots, r .
\end{aligned}
$$

Parameters $\tilde{c}_{k j}, \tilde{c}_{s j}, \tilde{a}_{i j}, \tilde{p}_{i}$, and $\tilde{B}$ in eq. (2.11) are fuzzy numbers. The fuzzy parameter model proposed by Li and Lee [8] may be summarized as follows: different ranges are used for maximization or minimization goals in eq. (2.11). For a maximization problem, $\left[f^{1}, f^{0}\right)$ type ranges may be used for the fuzzy coefficients in the goal function. On the other hand, fuzzy coefficients in the goal function must be set in $\left(f^{0}, f^{1}\right]$ range for the minimization problem. Then, the fuzzy coefficients $\tilde{a}_{i j}, \tilde{p}_{i}$ are $\left(f^{0}, f^{1}\right]$ type, and the fuzzy budget $\tilde{B}$ is $\left(f^{0}, f^{1}\right]$ type. Therefore, an optimal system design at the safety level $\alpha$ is formed according to the highest profit, lower cost, highest amount of 
available, lowest resources price, and smallest units of materials to be used for each product. Membership functions may be defined for these ranges using linear form. Next, the compromise solution is conducted for goal functions based on the defined $\alpha$-cut value. According to Li and Lee [8], $(x)_{\beta}^{\alpha}$ be a solution of $(2.2)$ where $\alpha \in[0 ; 1]$ denotes the possibility to which all fuzzy parameters satisfy, and $\beta \in[0 ; 1]$ denotes the compromise to which the solution satisfies the fuzzy goals at given possibility $\alpha$. $\lambda$ denotes the overall satisfaction for the solution $(x)_{\beta}^{\alpha}$. When $\alpha=\beta=\lambda$ the optimal solution is obtained. If the results do not satisfy $\alpha=\beta$, choose another $\alpha$ and solve phase I. The two-step approach [8] is described as follows. In the first step,

\section{$\operatorname{Max} \beta$}

Subject to

$$
\begin{aligned}
& \beta \leq\left[\sum_{j=1}^{n} \frac{\left(c_{k j}^{0}-\left(c_{k j}^{0}-c_{k j}^{1}\right) \alpha\right) x_{j}-\left(\tilde{Z}_{k}\right)_{\alpha}^{-}}{\left(\tilde{Z}_{k}\right)_{\alpha}^{*}-\left(\tilde{Z}_{k}\right)_{\alpha}^{-}}\right] \\
& \beta \leq\left[\sum_{j=1}^{n} \frac{\left.\left(\tilde{W}_{s}\right)_{\alpha}^{-}-\left(c_{s j}^{0}-\left(c_{s j}^{0}-c_{s j}^{1}\right) \alpha\right) x_{j}\right]}{\left(\tilde{W}_{s}\right)_{\alpha}^{-}-\left(\tilde{W}_{s}\right)_{\alpha}^{*}}\right] \\
& \sum_{i=1}^{m} \sum_{j=1}^{n} \frac{\left[p_{i}^{0}+\left(p_{i}^{1}-p_{i}^{0}\right) \alpha\right] \cdot\left[a_{i j}^{0}+\left(a_{i j}^{1}-a_{i j}^{0}\right) \alpha\right]}{B^{0}-\left(B^{0}-B^{1}\right) \alpha} \\
& x_{j} \geq 0, i=1,2, \ldots, m, j=1,2, \ldots, n, k=1,2, \ldots, l, \text { and } s=1,2, \ldots, r
\end{aligned}
$$

The second step model is formed as follows:

$$
\operatorname{Max} \bar{\beta}=\frac{1}{r+l}\left(\sum_{k=1}^{l} \beta_{k}+\sum_{s=1}^{r} \beta_{s}\right)
$$

Subject to

$$
\begin{aligned}
& \beta \leq \beta_{k} \leq \frac{\sum_{j=1}^{n}\left(c_{k j}^{o}-\left(c_{k j}^{o}-c_{k j}^{l}\right) \alpha \cdot x_{j}\right)-\left(\tilde{Z}_{k}\right)_{\alpha}^{-}}{\left(\tilde{Z}_{k}\right)_{\alpha}^{*}-\left(\tilde{Z}_{k}\right)_{\alpha}^{-}} \\
& \beta \leq \beta_{k} \leq \frac{\left(\tilde{W}_{s}\right)_{\alpha}^{-}-\sum_{j=1}^{n}\left(c_{k j}^{o}-\left(c_{k j}^{l}-c_{k j}^{0}\right) \alpha \cdot x_{j}\right)}{\left(\tilde{W}_{s}\right)_{\alpha}^{-}-\left(\tilde{W}_{s}\right)_{\alpha}^{*}} \\
& \sum_{i=1}^{m} \sum_{j=1}^{n}\left[p_{i}^{o}+\left(p_{i}^{l}-p_{i}^{o}\right) \alpha\right] a_{i j} x_{j} \leq B^{0}-\left(B^{0}-B^{l}\right) \alpha \\
& \beta_{k}, \beta_{s} \in[0,1], x_{j} \geq 0, k=1,2, \ldots, l, s=1,2, \ldots, r, \text { and } i=1,2, \ldots, m
\end{aligned}
$$


Analyzed by Li and Lee [8], in fuzzy environment based on de novo programming, model parameters and investment budget were made more flexible according to the features given below for the product mixture model:

a. When the constraint resources $\left(b_{i}\right)$ were constant, the fuzzy investment budget $(B)$ was determined using the fuzzy unit prices of resources $\left(\tilde{p}_{i}\right)$. In an investment budget like this one, the fuzzy investment budget was formed based only on the greatest tolerance value of unit prices.

b. It was estimated that the resource values that were needed to produce each product would decrease based on the smallest tolerance value, and the unit price for the resource amount of each product was evaluated based on the smallest acceptable unit price. In other words, the cost of resource amount was lowered in terms of resources and the lowest acceptable level of unit price of resource amount.

c. Finally, based on the order of goal function, if the goal function was maximization, goal coefficients were increased based on the tolerance value acceptable by the decision maker; and if goal function was minimization, those coefficients were decreased.

d. The model that was formed based on the factors above was solved according to the " $1-\alpha$ " risk protection (trust) level. When factors of risky and risk-free production are considered together, it must be determined in the beginning for which factors the decision maker would take risks. Then, the problem is to be modeled this way and solved according to an acceptable " $1-\alpha$ " risk protection level.

Rather than modeling the present production models at an acceptable risk level, the model should be formed to produce more efficiently by lowering risks in the beginning of product mixture problems. Based on de novo hypothesis, the increase or decrease of the budget will directly affect production as resource amounts are determined accordingly. Therefore, the increase or decrease of resource unit prices is significant for the budget. Additionally, the definition of resource amounts in a range will reform the budget. Consequently, the appropriate determination of both resource amounts and their unit prices results in a reasonable budget realization.

The risk factors in the studies model and assumptions on their analyses in fuzzy environment are listed as follows:

1. When allocating a budget for investment or resources, the constraint resources should be analyzed in a range based on the de novo programming. The budget is formed based on the constraint resource amounts. As the budget cannot exceed the acceptable tolerance value of constraint resources, there is less risk in terms of the budget, which is formed according to the expectations of the decision maker instead of surpassing. Nevertheless, businesses may only increase their budget up to a certain level. 
2. In the fuzzy de novo model proposed by Lee and Li [8], utilization amounts for products were decreased and unit prices were evaluated according to the smallest acceptable value and both of which increased production. On the other hand, the risk factors in production are lowered when the evaluation is based solely on unit prices as resource utilization amounts for each product are fixed. In other words, only the acceptable minimum value of unit prices should be taken into consideration when forming constraints in de novo models.

The proposed approach uses $\left[f^{1}, f^{0}\right)$ type range for fuzzy coefficients in maximizationtype goals and $\left(f^{0}, f^{1}\right]$ range for fuzzy coefficients in minimization-type goals. $\tilde{a}_{i j}$ coefficient is used as the constant number. The fuzzy coefficients $\tilde{p}_{i}$ and $\tilde{b}_{i}$ are of $\left(f^{0}, f^{1}\right]$ type, and the fuzzy budget $\tilde{B}$ is of $\left[f^{1}, f^{0}\right)$ type. It means that the optimal system design provides lowest cost, highest profit, lowest resource unit price, and highest budget utilization at $\alpha$ trust level. Consider the fuzzy de novo programming model based on the aforementioned information:

$$
\begin{aligned}
& \operatorname{Max} \tilde{Z}_{k}(x)=\sum_{j=1}^{n} \tilde{c}_{k j} x_{j} \\
& \operatorname{Min} \tilde{W}_{s}(x)=\sum_{j=1}^{n} \tilde{c}_{s j} x_{j}
\end{aligned}
$$

Subject to

$$
\begin{aligned}
& \sum_{j=1}^{n} a_{i j} x_{j}-b_{i} \leq 0 \\
& \sum_{i=1}^{m} \tilde{p}_{i} \tilde{b}_{i} \otimes \tilde{B} \\
& x_{j} \geq 0, \quad i=1,2, \ldots, m, j=1,2, \ldots, n, k=1,2, \ldots, l, \text { and } s=1,2, \ldots, r
\end{aligned}
$$

Solutions are determined as positive ideal or negative ideal for each goal function based on model (2.5):

$$
\begin{aligned}
& \forall k=1,2, \ldots, l \\
& j_{k}^{*}=\left\{j \in(1,2, \ldots, n): \frac{\max \left(\tilde{c}_{k j}\right)_{\alpha}^{U}}{\sum_{i=1}^{m}(p)_{\alpha}^{l} \cdot a_{i j}}\right\} \\
& x_{k}^{*}=\left\{\begin{array}{cc}
\frac{(\tilde{B})_{\alpha}^{U}}{\sum_{i=1}^{m}\left(\tilde{p}_{i}\right)_{\alpha}^{l} \cdot a_{i j}}, & j=j_{k}^{*} \\
0 & , \text { otherwise }
\end{array}\right.
\end{aligned}
$$




$$
\left(\tilde{Z}_{k}\right)_{\alpha}^{*}=\sum_{j=1}^{n}\left(\tilde{c}_{k j}\right)_{\alpha}^{U} \cdot x_{k}^{*}
$$

and

$$
\begin{aligned}
& j_{k}^{-}=\left\{j \in(1,2, \ldots, n): \frac{\min \left(\tilde{c}_{k j}\right)_{\alpha}^{L}}{\sum_{i=1}^{m}(p)_{\alpha}^{l} \cdot a_{i j}}\right\} \\
& x_{k}^{*}=\left\{\begin{array}{cl}
\frac{(\tilde{B})_{\alpha}^{U}}{\sum_{i=1}^{m}\left(\tilde{p}_{i}\right)_{\alpha}^{l} \cdot a_{i j}}, & j=j_{k}^{*} \\
0, & \text { otherwise }
\end{array}\right. \\
& \left(\tilde{Z}_{k}\right)_{\alpha}^{-}=\sum_{j=1}^{n}\left(\tilde{c}_{k j}\right)_{\alpha}^{U} \cdot x_{k}^{-} \\
& \forall s=1,2, \ldots, r \\
& j_{s}^{*}=\left\{j \in(1,2, \ldots, n): \frac{\min \left(\tilde{c}_{s j}\right)_{\alpha}^{L}}{\sum_{i=1}^{m}(p)_{\alpha}^{l} \cdot a_{i j}}\right\} \\
& x_{s}^{*}=\left\{\begin{array}{cc}
\frac{(\tilde{B})_{\alpha}^{U}}{\sum_{i=1}^{m}\left(\tilde{p}_{i}\right)_{\alpha}^{l} \cdot a_{i j}}, & j=j_{s}^{*} \\
0, & \text { otherwise }
\end{array}\right. \\
& \left(\tilde{W}_{s}\right)_{\alpha}^{*}=\sum_{j=1}^{n}\left(\tilde{c}_{s j}\right)_{\alpha}^{L} \cdot x_{k}^{*} \\
& j_{s}^{-}=\left\{j \in(1,2, \ldots, n): \frac{\max \left(\tilde{c}_{s j}\right)_{\alpha}^{U}}{\sum_{i=1}^{m}(p)_{\alpha}^{l} \cdot a_{i j}}\right\} \\
& x_{s}^{-}=\left\{\begin{array}{cc}
\frac{(\tilde{B})_{\alpha}^{U}}{\sum_{i=1}^{m}\left(\tilde{p}_{i}\right)_{\alpha}^{l} a_{i j}}, & j=j_{s}^{*} \\
0, & \text { otherwise }
\end{array}\right. \\
& \left(\tilde{W}_{s}\right)_{\alpha}^{-}=\sum_{j=1}^{n}\left(\tilde{c}_{s j}\right)_{\alpha}^{U} \cdot x_{k}^{-}
\end{aligned}
$$

Based on the " $\alpha$-cut" value determined by the decision maker, the compromise solution for goal functions is expressed as $\beta=\min \left\{\mu_{\tilde{Z}_{k}}^{\alpha}\left(Z_{k}\right), \mu_{\tilde{W}_{s}}^{\alpha}\left(W_{s}\right) ; k=1,2, \ldots, l\right.$, $s=1,2, \ldots, r\}$. The proposed model for these defined formulations is given below. It makes up the first step of the two-step approach: 
$\operatorname{Max} \beta$

Subject to

$$
\begin{aligned}
& \beta \leq\left[\frac{\sum_{j=1}^{n}\left(\tilde{c}_{k j}\right)_{\alpha}^{U} \cdot x_{j}-\left(\tilde{Z}_{k}\right)_{\alpha}^{-}}{\left(\tilde{Z}_{k}\right)_{\alpha}^{*}-\left(\tilde{Z}_{k}\right)_{\alpha}^{-}}\right] \\
& \beta \leq\left[\frac{\left(\tilde{W}_{s}\right)_{\alpha}^{-}-\sum_{j=1}^{n}\left(\tilde{c}_{s j}\right)_{\alpha}^{L} \cdot x_{j}}{\left(\tilde{W}_{s}\right)_{\alpha}^{-}-\left(\tilde{W}_{s}\right)_{\alpha}^{*}}\right] \\
& \sum_{i=1}^{m} \sum_{j=1}^{n}\left(\tilde{p}_{i}\right)_{\alpha}^{l} \cdot a_{i j} x_{j} \leq(\tilde{B})_{\alpha}^{U} \\
& x_{j} \geq 0, \alpha, \beta \in[0 ; 1] \\
& j=1,2, \ldots, n \text { and } s=1,2, \ldots, r
\end{aligned}
$$

After getting the optimal value of $\alpha$ and $\beta$, this solution is tested whether it is efficient or not. If eq. (2.11) is rewritten based on the membership functions of the parameters, the second step of the model is formed as follows:

$$
\operatorname{Max} \bar{\beta}=\frac{1}{r+l}\left(\sum_{k=1}^{l} \beta_{k}+\sum_{s=1}^{r} \beta_{s}\right)
$$

Subject to

$$
\begin{aligned}
& \beta \leq \beta_{k} \leq \frac{\sum_{j=1}^{n}\left(c_{k j}^{o}-\left(c_{k j}^{o}-c_{k j}^{l}\right) \alpha \cdot x_{j}\right)-\left(\tilde{Z}_{k}\right)_{\alpha}^{-}}{\left(\tilde{Z}_{k}\right)_{\alpha}^{*}-\left(\tilde{Z}_{k}\right)_{\alpha}^{-}} \\
& \beta \leq \beta_{k} \leq \frac{\left(\tilde{W}_{s}\right)_{\alpha}^{-}-\sum_{j=1}^{n}\left(c_{k j}^{o}-\left(c_{k j}^{l}-c_{k j}^{0}\right) \alpha \cdot x_{j}\right)}{\left(\tilde{W}_{s}\right)_{\alpha}^{-}-\left(\tilde{W}_{s}\right)_{\alpha}^{*}} \\
& \sum_{i=1}^{m} \sum_{j=1}^{n}\left[p_{i}^{o}+\left(p_{i}^{l}-p_{i}^{o}\right) \alpha\right] a_{i j} x_{j} \leq B^{0}-\left(B^{0}-B^{l}\right) \alpha \\
& x_{j} \geq 0, \beta \in[0 ; 1] \\
& k=1,2, \ldots, l, s=1,2, \ldots, r, \text { and } i=1,2, \ldots, m
\end{aligned}
$$

The optimal system design is achieved at $\lambda=\alpha=\beta$, where $\lambda$ represents the minimum level of possibilities under consideration of both the fuzzy goal and the fuzzy coefficients. 


\subsection{Illustrative example}

The multiobjective de novo programming problem prepared by Zeleny [23] was used to compare the results of the proposed approach and the approach by Li and Lee [8]. This problem consists of three maximization-type objective functions, six constraint functions, and three variables as follows:

$$
\begin{aligned}
& \operatorname{Max} Z_{1}=50 x_{1}+100 x_{2}+17.5 x_{3} \\
& \operatorname{Max} Z_{2}=92 x_{1}+75 x_{2}+50 x_{3} \\
& \operatorname{Max} Z_{3}=25 x_{1}+100 x_{2}+75 x_{3}
\end{aligned}
$$

Subject to

$$
\begin{aligned}
& 12 x_{1}+17 x_{2} \leq 1400 \\
& 3 x_{1}+9 x_{2}+8 x_{3} \leq 1,000 \\
& 10 x_{1}+13 x_{2}+15 x_{3} \leq 1,750 \\
& 6 x_{1}+16 x_{3} \leq 1325 \\
& 12 x_{2}+7 x_{3} \leq 900 \\
& 9.5 x_{1}+9.5 x_{2}+9.5 x_{3} \leq 1,075 \\
& x_{1}, x_{2}, x_{3} \geq 0
\end{aligned}
$$

The resource unit prices for $(\mathrm{P} 1)$ are $\$ 0.75, \$ 0.6, \$ 0.35, \$ 0.5, \$ 1.15, \$ 0.65$, respectively. The fuzzy parameter model for (P1) is as follows:

$$
\begin{aligned}
& \operatorname{Max} \tilde{Z}_{1}=\widetilde{50} x_{1}+100 x_{2}+17.5 x_{3} \\
& \operatorname{Max} \tilde{Z}_{2}=92 x_{1}+\widetilde{75} x_{2}+50 x_{3} \\
& \operatorname{Max} \tilde{Z}_{3}=\widetilde{25} x_{1}+100 x_{2}+75 x_{3}
\end{aligned}
$$

Subject to

$$
\begin{aligned}
& 12 x_{1}+17 x_{2} \leq \tilde{b}_{1} \\
& 3 x_{1}+9 x_{2}+8 x_{3} \leq 1,000 \\
& 10 x_{1}+13 x_{2}+15 x_{3} \leq \tilde{b}_{3} \\
& 6 x_{1}+16 x_{3} \leq 1325 \\
& 12 x_{2}+7 x_{3} \leq 900 \\
& 9.5 x_{1}+9.5 x_{2}+9.5 x_{3} \leq 1,075 \\
& \widetilde{0.75} \tilde{b}_{1}+0.6 \tilde{b}_{2}+\widetilde{0.35} \tilde{b}_{3}+0.5 \tilde{b}_{4}+\widetilde{1.15} \tilde{b}_{5}+0.65 \tilde{b}_{6} \leq \tilde{B} \\
& x_{1}, x_{2}, x_{3}, b_{1}, b_{2}, b_{3}, b_{4}, b_{5}, b_{6} \geq 0
\end{aligned}
$$


Defined as fuzzy triangular number, goal function coefficients are chosen for each goal function. They are $\widetilde{50}=(40,50,80), \widetilde{75}=(70,75,90)$, and $\widetilde{25}=(10,25,35)$. $\alpha$ - Cut information for the left side and right side corresponding to these coefficients are:

$$
\begin{aligned}
& (\widetilde{50})_{\alpha}^{L}=(40+10 \alpha),(\widetilde{50})_{\alpha}^{R}=(80-30 \alpha) \\
& (\widetilde{75})_{\alpha}^{L}=(70+5 \alpha), \quad(\widetilde{75})_{\alpha}^{R}=(90-15 \alpha) \\
& (\widetilde{25})_{\alpha}^{L}=(10+15 \alpha), \quad(\widetilde{25})_{\alpha}^{R}=(35-10 \alpha)
\end{aligned}
$$

$\alpha$-Cut information for the left side and the right side are organized as follows for a total of five fuzzy numbers such as two triangular fuzzy numbers accepted for the selected right side constants $\left(b_{i}\right)$, the two trapezoidal fuzzy numbers accepted for the selected unit prices $\left(p_{i}\right)$, and one triangular fuzzy number:

$$
\begin{aligned}
& \tilde{b}_{1}=\widetilde{1400}=(1,300,1,400,1,500) ; \\
& (\widetilde{1400})_{\alpha}^{L}=(1,300+100 \alpha),(\widetilde{14000})_{\alpha}^{R}=,(1500-100 \alpha) \\
& \tilde{b}_{3}=\widetilde{1750}=(1,500,1,750,2,000) ; \\
& (\widetilde{1750})_{\alpha}^{L}=(1,500+100 \alpha),(\widetilde{1750})_{\alpha}^{R}=(2,000-250 \alpha) \\
& \tilde{p}_{1}=\widetilde{0.75}=(0.6,0.7 .0 .8,1.15) ; \\
& (\widetilde{0.75})_{\alpha}^{L}=(0.6+0.1 \alpha),(\widetilde{0.75})_{\alpha}^{R}=(1.15-0.35 \alpha) \\
& \tilde{p}_{3}=\widetilde{0.35}=(0.3,0.35,0.5) ; \\
& (\widetilde{0.35})_{\alpha}^{L}=(0.3+0.05 \alpha),(\widetilde{0.35})_{\alpha}^{R}=(0.5-0.15 \alpha) \\
& \tilde{p}_{5}=\widetilde{1.15}=(1,1.15,1.2,1.4) ; \\
& (\widetilde{1.15})_{\alpha}^{L}=(1+0.15 \alpha),(\widetilde{1.15})_{\alpha}^{R}=(1.4-0.2 \alpha)
\end{aligned}
$$

Using this formulation, the calculation of the left side and the right side of the budget is as follows. For the left side of the constraint:

$$
\begin{aligned}
& (\widetilde{0.75})_{\alpha}^{L} *\left(12 x_{1}+17 x_{2}\right)+0.6^{*}\left(3 x_{1}+9 x_{2}+8 x_{3}\right)+(\widetilde{0.35})_{\alpha}^{L} *\left(10 x_{1}+13 x_{2}+15 x_{3}\right) \\
& +0.5^{\star}\left(6 x_{1}+16 x_{3}\right)+(\widetilde{1.15})_{\alpha}^{L} *\left(12 x_{2}+7 x_{3}\right)+0.65^{\star}\left(9.5 x_{1}+9.5 x_{2}+9.5 x_{3}\right.
\end{aligned}
$$

or 


$$
\begin{aligned}
= & (0.6+0.1 \alpha)^{\star}\left(12 x_{1}+17 x_{2}\right)+0.6^{\star}\left(3 x_{1}+9 x_{2}+8 x_{3}\right) \\
& +(0.3+0.05 \alpha)^{\star}\left(10 x_{1}+13 x_{2}+15 x_{3}\right) \\
& +0.5^{\star}\left(6 x_{1}+16 x_{3}\right)+(1+0.15 \alpha)^{\star}\left(12 x_{2}+7 x_{3}\right)+0.65^{\star}\left(9.5 x_{1}+9.5 x_{2}+9.5 x_{3}\right.
\end{aligned}
$$

is acquired. The final version of the calculation is

$$
(21.175+1.7 \alpha) x_{1}+(37.675+4,15 \alpha) x_{2}+(30.475+1,8 \alpha) x_{3}
$$

For the right side of the budget constraint:

$$
\begin{aligned}
\tilde{B}= & (1,500-100 \alpha)^{\star}(1.15-0.35 \alpha)+1,600^{\star} 0.6+(2,000-250 \alpha)^{\star}(0.5-0.15 \alpha) \\
& +1,325^{\star} 0.5+900^{\star}(1.4-0.2 \alpha)+1,075^{\star} 0.65 ; \tilde{B}=6,306.25-1,245 \alpha+40.5 \alpha^{2}
\end{aligned}
$$

Objective functions are organized as

$$
\begin{aligned}
& \operatorname{Max} \tilde{Z}_{1}=(80-30 \alpha) x_{1}+100 x_{2}+17.5 x_{3} \\
& \operatorname{Max} \tilde{Z}_{2}=92 x_{1}+(90-15 \alpha) x_{2}+50 x_{3} \\
& \operatorname{Max} \tilde{Z}_{3}=(75-50 \alpha) x_{1}+100 x_{2}+75 x_{3}
\end{aligned}
$$

(P1) is formed as below after these processes:

$$
\begin{aligned}
& \operatorname{Max} \tilde{Z}_{1}=(80-30 \alpha) x_{1}+100 x_{2}+17.5 x_{3} \\
& \operatorname{Max} \tilde{Z}_{2}=92 x_{1}+(90-15 \alpha) x_{2}+50 x_{3} \\
& \operatorname{Max} \tilde{Z}_{3}=(75-50 \alpha) x_{1}+100 x_{2}+75 x_{3}
\end{aligned}
$$

Subject to

$$
\begin{aligned}
&(21.175+1.7 \alpha) x_{1}+(37.675+4,15 \alpha) x_{2}+(30.475+1,8 \alpha) x_{3} \\
& \leq 6,306.25-1,245 \alpha+40.5 \alpha^{2} \\
& x_{1}, x_{2}, x_{3} \geq 0, \alpha \in[0,1]
\end{aligned}
$$

First, if a parametric solution is made for $\alpha=1$ (P3), it is obtained as follows:

$$
\begin{aligned}
& \operatorname{Max} Z_{1}=50 x_{1}+100 x_{2}+17.5 x_{3} \\
& \text { Max } Z_{2}=92 x_{1}+75 x_{2}+50 x_{3} \\
& \operatorname{Max} Z_{3}=25 x_{1}+100 x_{2}+75 x_{3}
\end{aligned}
$$

Subject to

$$
\begin{aligned}
& 22.875 x_{1}+41.825 x_{2}+32.275 x_{3} \leq 5,101.75 \\
& x_{1}, x_{2}, x_{3} \geq 0
\end{aligned}
$$

acquired. 
Positive ideal solutions for each objective function from (P3.1) are calculated as

$$
\left(\tilde{Z}_{1}\right)_{\alpha=1}^{*}=13,528.99 ;\left(\tilde{Z}_{2}\right)_{\alpha=1}^{*}=22,757.68 ;\left(\tilde{Z}_{3}\right)_{\alpha=1}^{*}=14,621.45
$$

and negative ideal solutions are calculated as

$$
\left(\tilde{Z}_{1}\right)_{\alpha=1}^{-}=0 ;\left(\tilde{Z}_{2}\right)_{\alpha=1}^{-}=0 ;\left(\tilde{Z}_{3}\right)_{\alpha=1}^{-}=0
$$

The first phase is written for (P3.1) using eq. (2.10) as follows:

$$
\operatorname{Max} \beta
$$

Subject to

$$
\begin{aligned}
& \beta \leq\left[\frac{50 x_{1}+100 x_{2}+17.5 x_{3}-0}{13,528.995-0}\right] \\
& \beta \leq\left[\frac{92 x_{1}+75 x_{2}+50 x_{3}-0}{22,757.68-0}\right] \\
& \beta \leq\left[\frac{25 x_{1}+100 x_{2}+75 x_{3}-0}{14,621.45-0}\right] \\
& 22.875 x_{1}+41.825 x_{2}+32.275 x_{3} \leq 5,101.75 \\
& x_{1}, x_{2}, x_{3} \geq 0, \beta \in[0 ; 1]
\end{aligned}
$$

Values obtained from the solution of (P3.2) are given in Table 2.1.

Table 2.1: Decision variables of Phase I for (P3.1).

\begin{tabular}{lccc}
\hline Decision variables & $Z_{\mathbf{3}}$ & $Z_{\mathbf{3}}$ & $Z_{\mathbf{3}}$ \\
\hline$x_{1}$ & 101.208 & 101.208 & 101.208 \\
\hline$x_{2}$ & 66.625 & 66.625 & 66.625 \\
\hline$x_{3}$ & 0 & 0 & 0 \\
\hline Objective function value & $11,722.9$ & $14,308.01$ & $10,457.8$ \\
\hline
\end{tabular}

Information in Table 2.1 is the result of the solution of (P3.1)'s first phase. The objective function value of (P3.1) is $\beta=0.6287$ that is for $\alpha=1, \beta=0.6287$. According to this result, the solution of (P3) will take place in the range of [0.6287; 1]. Because of $\alpha \neq \beta$, the solution should be continued by selecting a new $\alpha$. The result obtained for the selected $\alpha=0.71999$ is optimal. First, if (P3) is taken as $\alpha=0.71999$ 


$$
\begin{aligned}
& \operatorname{Max} Z_{1}=58.4 x_{1}+100 x_{2}+17.5 x_{3} \\
& \operatorname{Max} Z_{2}=92 x_{1}+79.2 x_{2}+50 x_{3} \\
& \operatorname{Max} Z_{3}=27.8 x_{1}+100 x_{2}+75 x_{3}
\end{aligned}
$$

Subject to

$$
\begin{aligned}
& 22.399 x_{1}+40.633 x_{2}+28.521 x_{3} \leq 5,430.845 \\
& x_{1}, x_{2}, x_{3} \geq 0
\end{aligned}
$$

is obtained. First, positive and negative ideal solutions for (P3.3) are given as follows:

$$
\left(\tilde{Z}_{1}\right)_{\alpha=0.71999}^{*}=14,159.62 ;\left(\tilde{Z}_{2}\right)_{\alpha=0.71999}^{*}=22,306.25 ;\left(\tilde{Z}_{3}\right)_{\alpha=0.71999}^{*}=14,281.17
$$

and

$$
\left(\tilde{Z}_{1}\right)_{\alpha=0.71999}^{-}=0 ;\left(\tilde{Z}_{2}\right)_{\alpha=0.71999}^{-}=0 ;\left(\tilde{Z}_{3}\right)_{\alpha=0.71999}^{-}=0
$$

Phase I is arranged using these solutions as follows:

Phase I

$$
\operatorname{Max} \beta
$$

Subject to

$$
\begin{aligned}
& 58.4 x_{1}+100 x_{2}+17.5 x_{3}-14,159.62 \beta \geq 0 \\
& 92 x_{1}+79.2 x_{2}+50 x_{3}-22,306.25 \beta \geq 0 \\
& 27.8 x_{1}+100 x_{2}+75 x_{3}-14,281.17 \beta \geq 0
\end{aligned}
$$

Subject to

$$
\begin{aligned}
& 22.399 x_{1}+40.633 x_{2}+28.521 x_{3} \leq 5,430.845 \\
& x_{1}, x_{2}, x_{3} \geq 0, \beta \in[0 ; 1]
\end{aligned}
$$

$\beta=0.71999, x_{1}=113.057869, x_{2}=71.332710, x_{3}=0$ are obtained from (P3.4)' solution.

In the first phase, $\beta=0.71999$ is obtained for $\alpha=0.71999$. It is now tested in phase 2 whether the solution of (P3.4) is efficient: 


$$
\operatorname{Max} \bar{\beta}=\frac{1}{3}\left(\beta_{1}+\beta_{2}+\beta_{2}\right)
$$

Subject to

$$
\begin{aligned}
& \beta \leq \beta_{1} \leq\left[\frac{58.4 x_{1}+100 x_{2}+17.5 x_{3}-0}{14,159.62-0}\right] \\
& \beta \leq \beta_{2} \leq\left[\frac{92 x_{1}+79.2 x_{2}+50 x_{3}-0}{22,306.25-0}\right] \\
& \beta \leq \beta_{3} \leq\left[\frac{27.8 x_{1}+100 x_{2}+75 x_{3}-0}{14,281.17-0}\right] \\
& 22.399 x_{1}+40.633 x_{2}+28.521 x_{3} \leq 5,430.845 \\
& \beta_{1} \geq 0.71999 \\
& \beta_{2} \geq 0.71999 \\
& \beta_{3} \geq 0.71999 \\
& x_{1}, x_{2}, x_{3} \geq 0, \beta \in[0 ; 1]
\end{aligned}
$$

Variable values obtained from (P3.5)'s solution created for phase 2 are specified as

$$
\begin{aligned}
& \bar{\beta}=0.80334, \beta_{1}=0.970072, \beta_{2}=0.71999, \beta_{3}=0.71999 \\
& x_{1}=113.057861, x_{2}=71.332710, \text { and } x_{3}=0
\end{aligned}
$$

This result shows that values obtained from phase 1 are efficient. Depending on the different values of $\alpha$, variable and objective function values are given in Table 2.2.

Table 2.2: The results of (P3).

\begin{tabular}{llllrr}
\hline$\alpha$ & $\beta$ & $Z_{1}$ & $Z_{2}$ & $Z_{3}$ & Budget \\
\hline 1.0 & 0.6973268 & 13002,15 & 15869.4 & 11598.99 & 5101.75 \\
0.75 & 0.7169044 & 12664.65 & 15584.99 & 11268.66 & 5395.281 \\
0.73 & 0.7191670 & 12743.9 & 15673.59 & 11341.23 & 5418,982 \\
0.725 & 0.7188874 & 12761.55 & 15718.16 & 11351,69 & 5424.913 \\
0.71999 & 0.71999 & 12786.05 & 15751.14 & 11372,84 & 5430.845 \\
\hline
\end{tabular}

The optimal result is obtained at $\alpha=\beta=0.71999$. At this point, resource utilization amounts according to decision variable values are 2569.328, 981.159, 2,057.886, 678.342, 855.984, and 1,751.696, respectively. 


\subsection{Conclusions}

As the main objective in traditional production processes is maximum profit and minimum production process, conciliatory or satisfactory solution is sought instead of optimal solution due to the multiple objective functions. In multiobjective de novo programming problems, it is possible to specify the optimal solutions at the end of similar processes. For example, if multiobjective de novo programming problem is solved through compromise programming, the result can be named as compromise optimal system design, or if it is solved through goal programming, the result can be named as satisfactory optimal system design. In this study, multiobjective de novo programming problem is solved because the solution of this problem is made in terms of fuzzy set theory, and is named as fuzzy optimal system design. However, the concept of compromise solution is used in some studies instead of fuzzy solution.

In this study, the model offered for fuzzy optimal system design comes into prominence with some features. First of them is although it is possible to define $a_{i j}$ parameters as a fuzzy number, it is wrong to fuzzify this parameter in the production process. This is because the change in the parameter $a_{i j}$ causes the amount used to produce the product during the production process and this disrupts the generated model. Also, $p_{i}$ and $b_{i}$ fuzzy parameters were used while setting a fuzzy budget. This situation caused the upper limit of the fuzzy $b_{i}$ determined by the decision maker to directly affect the budget while creating the fuzzy budget. The proposed approach can be accepted as an extension of Li and Lee's [8] approach because the solution procedure of the proposed approach is the same as that of $\mathrm{Li}$ and Lee's approach. Although it is possible to compare the results of Li and Lee's [8] approach with the result of the proposed approach, it is not recommended because of the differences explained earlier. Fuzzy parameters are different from each other, even though the solution of both approaches is the same.

\section{References}

[1] Li R.J. Multiple objective decision-making in a fuzzy environment, Yayınlanmamış Doktora Tezi, Kansas State University, 1990.

[2] Eiselt H.A. \& Sandblom C.L. Linear programming and its applications, Springer-Verlag Berlin Heidelberg, 2007.

[3] Yu P.L. Introduction to domination structures in multicriteria problems, In: Cochrane J.L. \& Zeleny M., eds., Multiple criteria decision making, University of South Carolina Press, Columbia SC, 1973.

[4] Mollaghasemi M. \& Pet-Edwards J. Technical briefing: making multiple- objective decisions, IEEE Computer Society Press Los Alamitos, California, 1997.

[5] Zimmermann H.J. (1978) Fuzzy programming and linear programming with several functions, Fuzzy Sets and Sysytems, 1, 45-55. 
[6] Zeleny M. Multiobjective Design of High-Productivity Systems, Proceedings of Joint. Automatic Control Conference, July 27-30, Purdue University.1976.

[7] Zeleny M. (1981) On the squanering of resources and profits via linear programming, Interfaces, 11(5), 101-107.

[8] Li R.J. \& Lee E.S. (1993) Fuzzy multiple objective programming and compromise programming with pareto optimum, Fuzzy Sets and Systems, 53, 275-288.

[9] Zeleny M. Systems Approach to Multiple Criteria Decision Making: Metaoptimum, In: Toward interactive and intelligent decision support systems, edited by, Sawaragi Y., Inoue K., \& Nakayama H., Springer-Verlag, New York, 1987, 28-37.

[10] Zeleny M. (1990) Optimizing given systems vs. designing optimal systems: the De Novo programming approach, International Journal of General Systems, 17(4), 295-307.

[11] Zeleny M. Multicriterion Design of High-Productivity Systems: Extensions and Applications, In: Decision making with multiple objectives, edited by, Haimes Y.Y. \& Chankong V., Springer-Verlag, New York, 1985, 308-321.

[12] Hessel M. \& Zeleny M. (1987) Optimal system design: towards new interpretation of shadow prices in linear programming, Computer Operational Research, 14(4), 265-271.

[13] Shi Y. (1995) Studuies on optimum-path ratios in multicriteria De Novo programming problems, Computers Mathamatical Applications, 29(5), 43-50.

[14] Umarusman N. (2013) Min-max goal programming approach for solving multi-objective de novo programming problems, International Journal of Operations Research, 10(2), 92-99.

[15] Zhuang Z.Y. \& Hocine A. (2018) Meta goal programing approach for solving multi-criteria de Novo programming problem, European Journal of Operational Research, 265(1), 228-238.

[16] Lai Y.-J. \& Hwang C.-L. Fuzzy mathematical programming; methods and applications (First edition), Berlin Heidelberg, Springer-Verlag, 1992.

[17] Li R.J. \& Lee E.S. (1990a) Approaches to multicriteria de novo programs, Journal of Mathematical Analysis and Applications, 153, 97-111.

[18] Li R.J. \& Lee E.S. (1990b) Multi-criteria de novo programming with fuzzy parameters, Computers Mathamatical Applications, 19(56), 13-20.

[19] Bhattacharya D. \& Chakraborty S. (2018) Solution of the general multi-objective De-Novo programming problem using compensatory operator under fuzzy environment, Journal of Physics: Conference Series, 1039(1), 1-8.

[20] Umarusman N. (2018) A fuzzy approach proposal in the solution of multi objective De Novo programming problems and a business application, Business and Economics Research Journal, 9(4), 825-838.

[21] Zeleny M. Linear multiobjective programming, Springer-Verlag, New York, 1974.

[22] Zadeh L.A. (1965) Fuzzy Sets, Information and Control, 8, 338-353.

[23] Zeleny M. (1986) An external reconstruction approach (ERA) to linear programming, Computers and Operations Research, 13(1), 95-100.

[24] Babic Z. \& Pavic I. (1999) Multicriterial production planning by De Novo programming approach, International Journal of Production Economics, 43, 59-66.

[25] Tabucanon M.T. Multiple criteria decision making in industry, Elsevier, New York, 1998.

[26] Bhattacharya D. \& Banik S. (2019) Hacettepe University Bulletin of Natural Sciences and Engineering Series B: Mathematics and Statistics, 49(1), 1-14. 
